work is required to develop oesophageal examination techniques using MACE.

\section{PTU-121 THE VALUE OF ENDOSCOPIC BIOPSIES IN ROUTINE UPPER GASTROINTESTINAL ENDOSCOPY}

David Tai*, Mustafa Jalal, Gloria Tun, Stefania Chetcuti-Zammit, Mark McAlindon. Sheffield Teaching Hospitals, Sheffield, UK

\subsection{6/gutjnl-2019-BSGAbstracts.110}

Introduction The BSG and AUGIS quality standards in upper gastrointestinal endoscopy recommend taking biopsies for histological examination to diagnose inflammatory, pre- or malignant lesions which may or may not be visible to the naked eye [Beg, Gut 2018]. This may increase the duration and cost of the procedure and potentially reduce the focus on mucosal inspection. This study aimed to investigate the diagnostic value (changes in diagnosis and contribution to management) added by histology and its cost.

Methods Patients undergoing consecutive diagnostic OGDs performed by four fellows were recruited. Incomplete, surveillance or therapeutic procedures, procedures for dysphagia and those arranged in order to obtain histology (eg. positive coeliac serology) were excluded. Biopsies were performed according to BSG quality standards [Beg, Gut 2018]. The utility of biopsies (changes in diagnosis and contribution to management) were examined from endoscopic and histological findings.

Results Of 509 patients examined, 314 were included. OGD was performed for dyspepsia (66\%), anaemia (26\%), weight loss (16\%), diarrhoea (2\%) and vomiting (1\%). Biopsies occurred in $86 \%$ of patients with an mean of 1.5 samples per patient biopsied. Biopsy sampling provided additional pathological information not evident at endoscopy in $28 \%$ of cases. However, if benign pathologies are excluded (mostly chemical or Helicobacter gastritis or duodenitis), this falls to $6.7 \%$ $(n=18$ : dysplasia $n=3$, adenocarcinoma $n=1$, gastric intestinal metaplasia (GIM) $n=7$, villous atrophy (VA) $n=2$, Barretts $\mathrm{n}=5$ ). In total, management change occurred in $19 \%$ consequent on histological findings, but this falls to $12 \% \quad(n=34$ : adenocarcinoma $\mathrm{n}=2$, dysplasia $\mathrm{n}=3$, GIM, $\mathrm{n}=10$, Barretts $\mathrm{n}=15$, VA $\mathrm{n}=3$, peptic stricture $\mathrm{n}=1$ ) where Helicobacter pylori (which can be detected by other non-invasive methods) eradication is excluded.

Fifty seven focal lesions were biopsied where dysplasia or cancer was diagnosed in 7\% (1/14 oesophageal, 2/35 gastric and $1 / 8$ duodenal). Barretts, GIM and VA were found more commonly in abnormal compared to normal mucosa (See table 1). The overall cost of tissue sampling approximates to $£ 17,100$ ( $£ 82$ per person). Routine biopsies of patients with only normal mucosa costed over $£ 3,500$.

Abstract PTU-121 Table 1 Diagnostic yield for premalignant conditions in abnormal vs normal mucosa

\begin{tabular}{llll}
\hline & Abnormal & Normal & $\mathrm{p}$ \\
\hline Barrett's & $\%(\mathrm{n})$ & & \\
GIM & $58 \%(14)$ & $0 \%(0)$ & 0.02 \\
Villous atrophy & $81 \%(9)$ & $18 \%(2)$ & 0.05 \\
\hline
\end{tabular}

Conclusions Although 86\% undergoing OGD have biopsies taken, contributing to aetiopathological understanding in $28 \%$, only a small minority $(6.7 \%)$ have pre-malignant or malignant pathologies and result in a change in management in only $12 \%$. Non-targeted biopsies are of limited value and costly when sampled from normal mucosa.

\section{PTU-122 A PILOT DATA IN UNDERSTANDING THE CAUSES \& TEMPORAL RELATIONSHIP OF ELECTIVE ENDOSCOPY UNPLANNED ADMISSIONS WITHIN 8DAYS}

Bee Theng Tan*, Irfan Amin. Peterborough City Hospital, Peterborough, UK

\subsection{6/gutjnl-2019-BSGAbstracts.111}

Introduction We aim to understand the causes and temporal relationship of unplanned admissions within 8 days of elective endoscopy as per JAG recommendation.

Background We retrospectively examined all unplanned admissions within 8 days of elective endoscopy from Jan-June 2018. Demographic and co-morbidity data as defined by Charlson Comorbidity Index (CCI) and causes of admissions were collected.

Unplanned admissions were divided into endoscopic related and non-endoscopic related causes. The former was further divided into direct (perforation, infection, bleeding, and postpolypectomy syndrome) and indirect (sedation-related, displaced NG/NJ tube, or abdominal pain) complications.

Admissions were then classified as early (Day 0-2) or late (Day 3-8). Data was collected using Microsoft Excel and analyzed for any association between endoscopic complications with admission time.

Results Of the 5482 elective endoscopy patients, 65(1.18\%) had unplanned admissions within 8 days. The majority 62/65 (95\%) had 1 or more co-morbidities (CCI score $\geq 1$ ). High CCI scores may thus be a risk factor for unplanned admissions. Of all endoscopic procedures, ERCP had the highest admissions rate. 12 of $164(7.3 \%)$ patients undergoing elective ERCP had unplanned admissions. Colonoscopy and sigmoidoscopy had the lowest unplanned admission rate at $12 / 2038$ $(0.5 \%)$ and $4 / 788(0.5 \%)$ respectively.

Of the 65 unplanned admissions, 26/65(40\%) were admitted within the first 72 hours and 39/65(60\%) thereafter. Most early admissions $(14 / 26=54 \%)$ were related to endoscopic complications while the remaining were due to a non-endoscopic cause $(12 / 26=46 \%)$.

In contrast, the majority of late admissions were related to non-endoscopic causes $(31 / 39=80 \%)$. Endoscopy related complications in this timeframe were minimal $(8 / 39=20 \%)$.

Overall, ERCP has the highest risk of direct $(8 / 164=4.8 \%)$ and indirect complications $(2 / 164=1.2 \%)$ amongst the unplanned admissions. In addition, ERCP carries a significantly higher risk of direct than indirect complications $(p=0.0361)$. This may therefore explain the highest unplanned admissions rate found in ERCP compared to other endoscopic procedures.

Summary Our study provides crude data analyzing the risk of unplanned admissions related to elective endoscopic procedures in a busy DGH endoscopy unit. We aimed to understand if there was any temporal relationship in unplanned admissions post-elective endoscopy. Subsequent data demonstrated majority of direct complications of endoscopy were admitted within the first 72 hours with ERCP carrying the 


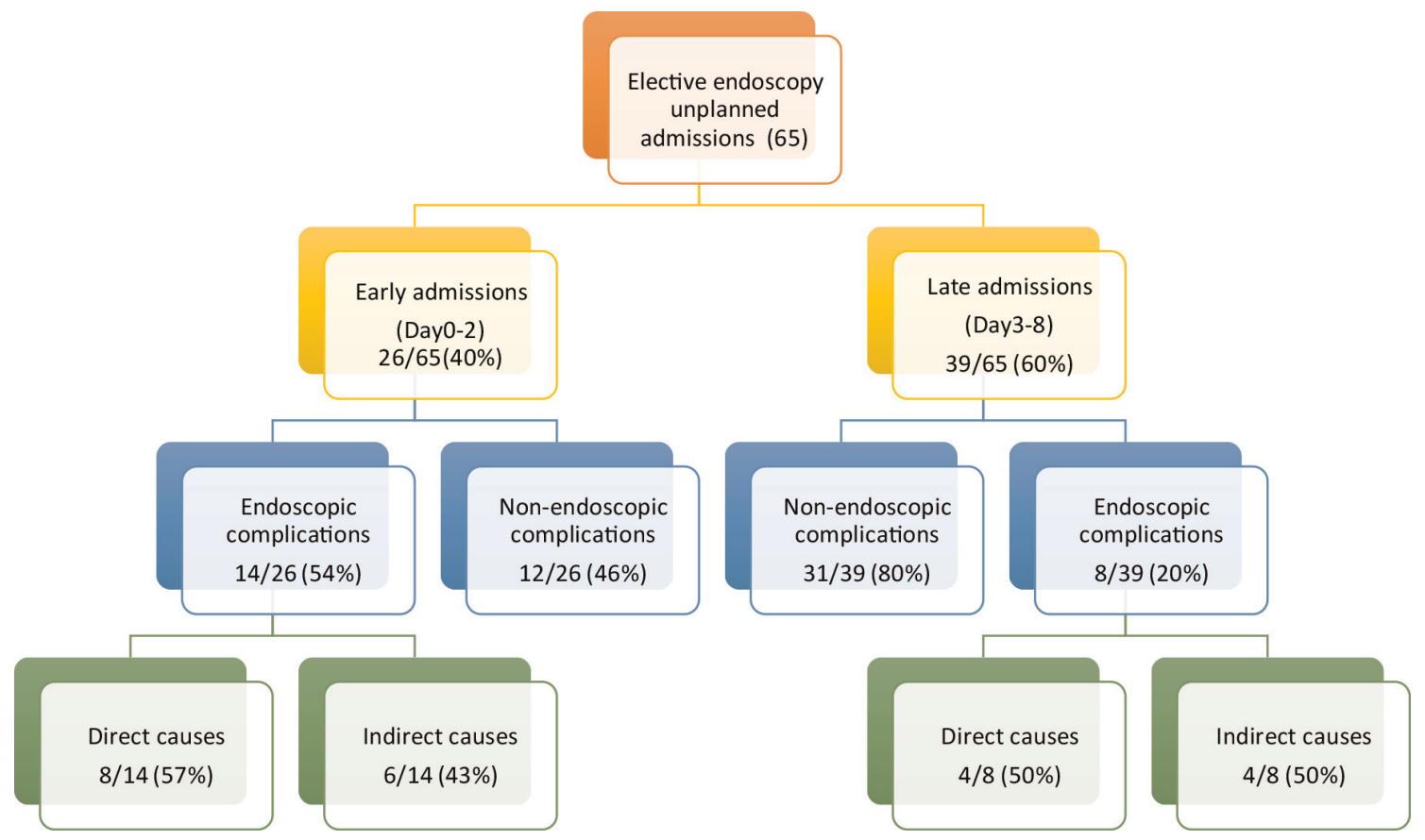

Abstract PTU-122 Figure 1 Early vs late unplanned admissions

highest risk. However, more studies are needed to see if these results are reproducible and related to individual procedure. Early endoscopy readmissions could therefore be used as a surrogate marker for endoscopic complications and has the potential to be used in a KPI study.

\section{PTU-123 ENDORINGS ASSISTED COLONOSCOPY VERSUS STANDARD COLONOSCOPY FOR POLYP DETECTION: A RANDOMISED CONTROLLED TRIAL}

${ }^{1}$ Sreedhari Thayalasekaran*, ${ }^{1}$ Rupam Bhattacharyya, ${ }^{2}$ Fergus Chedgy, ${ }^{3}$ Peter Basford, ${ }^{1}$ Asma Alkandari, 'Sharmila Subramaniam, 'Kesavan Kandiah, 'Fergus Thursby-Pelham, ${ }^{1}$ Richard Ellis, 'Sergio Coda, 'Patrick Goggin, 'Gaius Longcroft-Wheaton, ${ }^{1}$ Pradeep Bhandari. ${ }^{1}$ Queen Alexandra Hospital, Portsmouth, UK; ${ }^{2}$ Royal Sussex County Hospital, Brighton, UK; ${ }^{3}$ St Richards Hospital, Chichester, UK

\subsection{6/gutjnl-2019-BSGAbstracts.112}

Introduction The Endorings ${ }^{\mathrm{TM}}$ is a distal attachment consisting of two layers of circular flexible rings that evert mucosal folds. Aims; to investigate if Endorings Colonoscopy (ER) improves polyp and adenoma detection compared to standard colonoscopy (SC).

Methods This multi-centre, parallel group, randomized controlled trial included screening, surveillance and symptomatic patients. Primary outcome; number of polyps per patient. Secondary outcomes; number of adenomas per patient, adenoma/ polyp detection rates and withdrawal times.

Results Total of 556 patients (214 females, 342 males) randomized to ER (275) or SC (281). Mean age 67. Colonoscopy completed 532/556 (96\%) cases. EndoRings removed in $74 / 275(27 \%)$ patients. In $66 / 74(89 \%)$ cases removal was performed due to difficulties with sigmoid intubation. Remainder removed to facilitate retroflexion or polyp removal/retrieval. Total number of polyps in ER limb 571 vs 444 in SC limb. Total number of adenomas in ER limb 361 vs 343 for SC limb. Our study showed a statistically significant difference in the mean number of polyps per patient in both the Intention to Treat (ITT) (1.8 SC vs $2.1 \mathrm{ER}$, p-value $0.02)$ and Per Protocol (PP) (1.8 SC vs 2.25 ER, p-value $0.009)$.

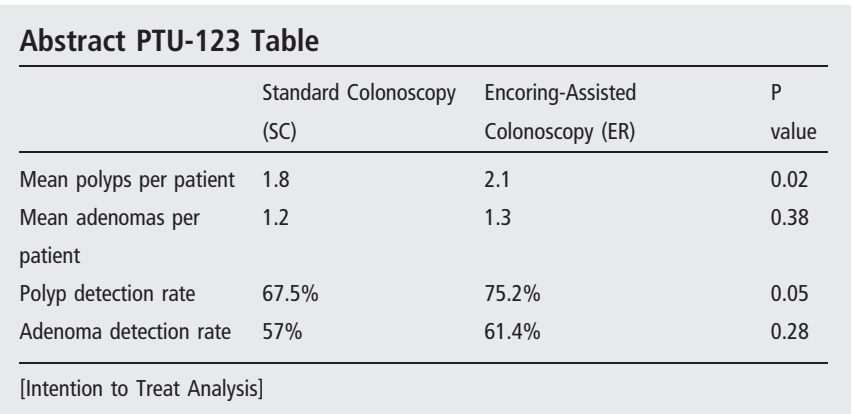

There was a trend towards a greater polyp detection rate in the ER colonoscopy $(67.5 \%$ SC vs $75.2 \%$ ER, p-value $0.05)$.

Conclusions Despite the high removal rate of Endorings, there was a statistically significant increase in the mean number of polyps in the ER limb compared to the SC limb. Our study shows promise for the EndoRings ${ }^{\mathrm{TM}}$ device to improve polyp detection.

\section{PTU-124 INCREASED DIAGNOSTIC SENSITIVITY OF PANCREATICOBILIAY MALIGNANCY BY MODIFYING ERCP BRUSHING PRACTICE: A SINGLE CENTRE EXPERIENCE}

Ioannis Varmpompitis*, Khansa Adam, Jonathan Booth, Sinead McEvoy, Nishchay Chandra. Royal Berkshire Hospital, Nea Smirni, Athens, Greece

10.1136/gutjnl-2019-BSGAbstracts.113 